\title{
Fast elliptic-curve cryptography on the Cell Broadband Engine
}

\author{
Neil Costigan ${ }^{1}$ and Peter Schwabe ${ }^{2}$ * \\ 1 School of Computing \\ Dublin City University, Glasnevin, Dublin 9, Ireland \\ neil.costigan@computing.dcu.ie \\ 2 Department of Mathematics and Computer Science \\ Technische Universiteit Eindhoven, P.O. Box 513, 5600 MB Eindhoven, Netherlands \\ peter@cryptojedi.org
}

\begin{abstract}
This paper is the first to investigate the power of the Cell Broadband Engine for state-of-the-art public-key cryptography. We present a high-speed implementation of elliptic-curve Diffie-Hellman (ECDH) key exchange for this processor, which needs 777000 cycles on one Synergistic Processor Unit for a scalar multiplication on a 255-bit elliptic curve, including the costs for key verification and key compression. This cycle count is independent of inputs therefore protecting against timing attacks.

This speed relies on a new representation of elements of the underlying finite field suited for the unconventional instruction set of this architecture.

Furthermore we demonstrate that an implementation based on the multiprecision integer arithmetic functions provided by IBM's multi-precision math (MPM) library would take at least 2227040 cycles.

Comparison with implementations of the same function for other architectures shows that the Cell Broadband Engine is competitive in terms of cost-performance ratio to other recent processors such as the Core 2 for public-key cryptography.

Specifically, the state-of-the-art Galbraith-Lin-Scott ECDH software performs 27370 scalar multiplications per second using all four cores of a 2.5GHz Intel Core 2 Quad Q9300 inside a $\$ 400$ computer, while the new software reported in this paper performs 24528 scalar multiplications per second on a Playstation 3 that costs just $\$ 279$. Both of these speed reports are for high-security 256-bit elliptic-curve cryptography.
\end{abstract}

Keywords: Cell Broadband Engine, elliptic-curve cryptography (ECC), efficient implementation

\footnotetext{
* The first author was supported by the Irish Research Council for Science, Engineering and Technology (IRCSET). The second author was supported by the European Commission through the ICT Programme under Contract ICT-2007-216499 CACE, and through the ICT Programme under Contract ICT-2007-216646 ECRYPT II. Permanent ID of this document: a33572712a64958c0bf522e608f25f0d. Date: Jan 21, 2009
} 


\section{Introduction}

This paper describes a high-speed implementation of state-of-the-art public-key cryptography for the Cell Broadband Engine (CBE). More specifically we describe an implementation of the curve25519 function, an elliptic-curve DiffieHellman key exchange (ECDH) function introduced in [3].

Implementations of this function have been achieving speed records for highsecurity ECDH software on different platforms [3], [9]. Benchmarks of our implementation show that the $\mathrm{CBE}$ is competitive (in terms of cost-performance ratio) to other recent processors as the Intel Core 2 for public-key cryptography.

Our implementation needs 777000 cycles on one Synergistic Processor Unit (SPU) of the CBE. This number includes not only scalar multiplication on the underlying 255-bit elliptic curve, but furthermore key compression, key validation and protection against timing attacks. We plan to put our implementation into the public domain to maximize reusability of our results.

We wish to thank Dan Bernstein, Tanja Lange, Ruben Niederhagen, and Michael Scott for their invaluable feedback and encouragement. Neil Costigan would also like to thank Luleå University of Technology, Sweden.

\subsection{How these speeds were achieved}

Elliptic-curve cryptography (ECC) is usually implemented as a sequence of arithmetic operations in a finite field. IBM provides a multi-precision math (MPM) library developed especially for the Cell Broadband Engine as part of the standard Cell Software Development Kit (SDK). The obvious approach for the implementation of ECC on the CBE is thus to use this library for the underlying finite field arithmetic.

However, we will show that the targeted performance cannot be achieved following this approach, not even with optimizing some functions of the MPM library for arithmetic in fields of the desired size.

Instead, the speed of our implementation is achieved by

- Parting with the traditional way of implementing elliptic-curve cryptography which uses arithmetic operations in the underlying field as smallest building blocks,

- Representing finite field elements in a way that takes into account the special structure of the finite field and the unconventional SPU instruction set, and

- Careful optimization of the code on assembly level.

Related work Implementations of public-key cryptography for the Cell Broadband Engine have not yet been extensively studied. In particular we don't know of any previous implementation of ECC for the Cell Broadband Engine.

Costigan and Scott investigate in [5] the use of IBM's MPM library to accelerate OpenSSL on a Sony Playstation 3. The paper reports benchmarks for 
RSA with different key lengths; RSA signature generation with a 2048 bit key is reported to take $0.015636 \mathrm{~s}$ corresponding to 50035200 cycles on one SPU.

An implementation of the Digital Signature Algorithm (DSA) supporting key lengths up to 1024 bits is included in the SPE Cryptographic Library [15].

In [17] Shimizu et al. report 4074000 cycles for 1024-bit-RSA encryption or decryption and 1331000 cycles for 1024-bit-DSA key generation. Furthermore they report 2250000 cycles for 1024-bit-DSA signature generation and 4375000 cycles for 1024-bit-DSA signature verification.

The Cell Broadband Engine has recently demonstrated its power for cryptanalysis of symmetric cryptographic primitives [19], [18],

Organization of the paper In Section 2 we will briefly review the features of the $\mathrm{CBE}$ which are relevant for our implementations. Section 3 describes the curve25519 function including some necessary background on elliptic-curve arithmetic. Section 4 describes IBM's MPM library including optimizations we applied to accelerate arithmetic in finite fields of the desired size. We show that an implementation based on this library cannot achieve the targeted performance. In Section 5 we detail our implementation of curve25519. We conclude the paper with a discussion of benchmarking results and a comparison to ECDH implementations for other architectures in Section 6.

\section{The Cell Broadband Engine}

When it became apparent that multi-core chip design rather than increased frequency was the gateway to more efficient CPUs, IBM, Sony and Toshiba took a novel approach: Instead of developing a chip with every core being the same, they came up with the Cell Broadband Engine Architecture (CBEA). Currently two different CBEA processors are available: the Cell Broadband Engine (CBE) and the PowerXCell $8 \mathrm{i}$ processor. Both are multi-core processors consisting of a traditional central processor and 8 specialized high performance processors called Synergistic Processor Units (SPUs).

These units are combined across a high bandwidth (204 GB/s) [11] bus to offer a multi-core environment with two instruction sets and enormous processing power. Compared with the CBE the PowerXCell 8i processor has highly increased double precision floating point performance. The implementation described in this paper is optimized for the CBE, we will therefore in the following focus on the description of this processor.

The Cell Broadband Engine can be found in the Sony Playstation 3 and the IBM QS20 and QS21 blade server series. Note that the CBE in the Playstation 3 makes just 6 out of 8 SPUs available for general purpose computations. Toshiba equips several laptops of the Qosmio series with the SpursEngine consisting of 4 SPUs intended for media processing. This SpursEngine can also be found in a PCI Express card called WinFast pxVC1100 manufactured by Leadtek which is currently available only in Japan.

The primary processor of the Cell Broadband Engine is a 64-bit Power Processor Unit (PPU). This PPU works as a supervisor for the other cores. Currently 
operating at $3.2 \mathrm{GHz}$, The PPU is a variant of the G5/PowerPC product line, a RISC driven processor found in IBM's servers and Apple's PowerMac range.

\subsection{The Cell's SPU}

The real power of the CBE is in the additional SPUs. Each SPU is a specialist processor with a RISC-like SIMD instruction set and a 128-element array of 128bit registers. It has two pipelines (pipeline 0 and pipeline 1); each cycle it can dispatch one instruction per pipeline. Whether or not the SPU really dispatches two instructions in a given cycle is highly dependent on instruction scheduling and alignment. This is subject to the following conditions:

- Execution of instructions is purely in-order.

- The two pipelines execute disjoint sets of instructions (i.e. each instruction is either a pipeline-0 or a pipeline-1 instruction).

- The SPU has a fetch queue that can contain at most two instructions.

- Instructions are fetched into the fetch queue only if the fetch queue is empty.

- Instructions are fetched in pairs; the first instruction in such a pair is from an even word address, the second from an odd word address.

- The SPU executes two instructions in one cycle only if two instructions are in the fetch queue, the first being a pipeline-0 instruction and the second being a pipeline-1 instruction and all inputs to these instructions being available and not pending due to latencies of previously executed instructions.

Hence, instruction scheduling has to ensure that pipeline-0 and pipeline-1 instructions are interleaved and that latencies are hidden; instruction alignment has to ensure that pipeline- 0 instructions are at even word addresses and pipeline1 instructions are at odd word addresses.

Both our implementation and the MPM library build the finite field arithmetic on the integer arithmetic instructions of the SPU. This is due to the fact that single-precision floating-point arithmetic offers a too small mantissa and that double-precision floating-point arithmetic causes excessive pipeline stalls on the SPU and is therefore very inefficient.

All integer arithmetic instructions (except shift and rotate instructions) are SIMD instructions operating either on 4 32-bit word elements or on 8 16-bit halfword elements or on 16 8-bit byte elements of a 128-bit register.

Integer multiplication constitutes an exception to this scheme: The integer multiplication instructions multiply 4 16-bit halfwords in parallel and store the 32 -bit results in the 4 word elements of the results register.

The following instructions are the most relevant for our implementation; for a detailed description of the SPU instruction set see [13], for a list of instruction latencies and associated pipelines see [12, Appendix B].

a: Adds each 32-bit word element of a register $a$ to the corresponding word element of a register $b$ and stores the results in a register $r$. 
mpy: Multiplies the 16 least significant bits of each 32-bit word element of a register $a$ with the corresponding 16 bits of each word element of a register $b$ and stores the resulting four 32-bit results in the four word elements of a register $r$.

mpya: Multiplies 16-bit halfwords as the mpy instruction but adds the resulting four 32-bit word elements to the corresponding word elements of a register $c$ and stores the resulting sum in a register $r$.

shl: Shifts each word element of a register $a$ to the left by the number of bits given by the corresponding word element of a register $b$ and stores the result in a register $r$.

rotmi: Shifts of each word element of a register $a$ to the right by the number of bits given in an immediate value and stores the result in a register $r$.

shufb: Allows to set each of the result register $r$ to either the value of an arbitrary byte of one of two input registers $a$ and $b$ or to a constant value of $0,0 x 80$ or $0 x f f$.

\subsection{Computation micro-kernels for the SPU}

Alvaro, Kurzak and Dongarra [1] introduce the idea of a computation microkernel for the SPU where the restricted code and data size of the SPU become important design criteria but issues such as inter-chip communication and synchronization are not considered. The kernel focuses on utilization of the wide registers and the instruction level parallelism. Furthermore, for security aware applications such as those using ECC, there is an interesting security architecture where an SPU can run in isolation mode, where inter-chip communications, loading and unloading program code incur significant overhead. In this paper we describe such a computation micro-kernel implementation running on one SPU of the CBE.

\section{ECDH and the curve25519 function}

\subsection{Elliptic-Curve Diffie-Hellman Key Exchange (ECDH)}

Let $\mathbb{F}$ be a finite field and $E / \mathbb{F}$ an elliptic curve defined over $\mathbb{F}$. Let $E(\mathbb{F})$ denote the group of $\mathbb{F}$-rational points on $E$. For any $P \in E(\mathbb{F})$ and $k \in \mathbb{Z}$ we will denote the $k$-th scalar multiple of $P$ as $[k] P$.

The Diffie-Hellman key exchange protocol [6] can now be carried out in the group $\langle P\rangle \subseteq E(\mathbb{F})$ as follows: User $A$ chooses a random $a \in\{2, \ldots,|\langle P\rangle|-$ $1\}$, computes $[a] P$ and sends this to user $B$. User $B$ chooses a random $b \in$ $\{2, \ldots,|\langle P\rangle|-1\}$, computes $[b] P$ and sends this to user $A$. Now both users can compute $Q=[a]([b] P)=[b]([a] P)=[(a \cdot b)] P$. The joint key for secret key cryptography is then extracted from $Q$; a common way to do this is to compute a hash value of the $x$-coordinate of $Q$. 


\subsection{Montgomery Arithmetic}

For elliptic curves defined by an equation of the form $B y^{2}=x^{3}+A x^{2}+x$, Montgomery introduced in [16] a fast method to compute the $x$-coordinate of a point $R=P+Q$, given the $x$-coordinates of two points $P$ and $Q$ and the $x$-coordinate of their difference $P-Q$.

These formulas lead to an efficient algorithm to compute the $x$-coordinate of $Q=[k] P$ for any point $P$. This algorithm is often referred to as the Montgomery ladder. In this algorithm the $x$-coordinate $x_{P}$ of a point $P$ is represented as $\left(X_{P}, Z_{P}\right)$, where $x_{P}=X_{P} / Z_{P}$; for the representation of the point at infinity see the discussion in Appendix B of [3]. See Algorithms 1 and 2 for a pseudocode description of the Montgomery ladder.

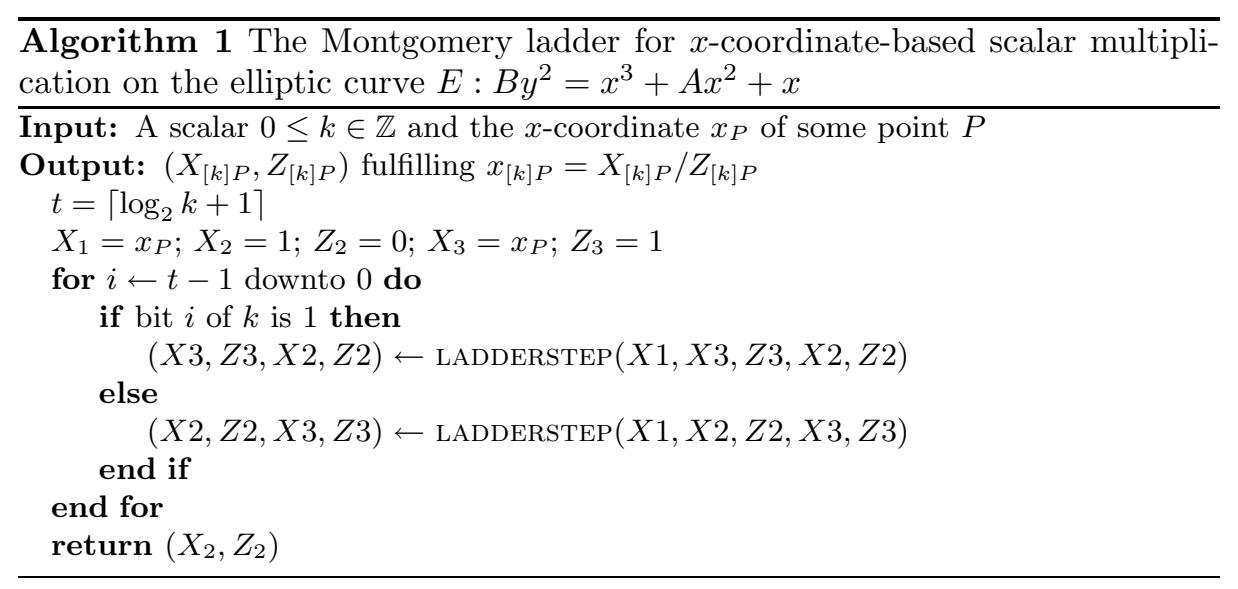

Each "ladder step" as described in Algorithm 2 requires 5 multiplications, 4 squarings, 8 additions and one multiplication with the constant $a 24=(A+2) / 4$ in the underlying finite field.

\subsection{The curve25519 function}

Bernstein proposed in [3] the curve25519 function for elliptic-curve Diffie-Hellman key exchange. This function uses arithmetic on the elliptic curve defined by the equation $E: y^{2}=x^{3}+A x^{2}+x$ over the field $\mathbb{F}_{p}$, where $p=2^{255}-19$ and $A=486662$; observe that this elliptic curve allows for the $x$-coordinate-based scalar multiplication described above.

The elliptic curve and underlying finite field are carefully chosen to meet high security requirements and to allow for fast implementation, for a detailed discussion of the security properties of curve25519 see [3].

The curve25519 function takes as input two 32-byte strings, one representing the $x$-coordinate of a point $P$ and the other representing a 256-bit scalar $k$. It 


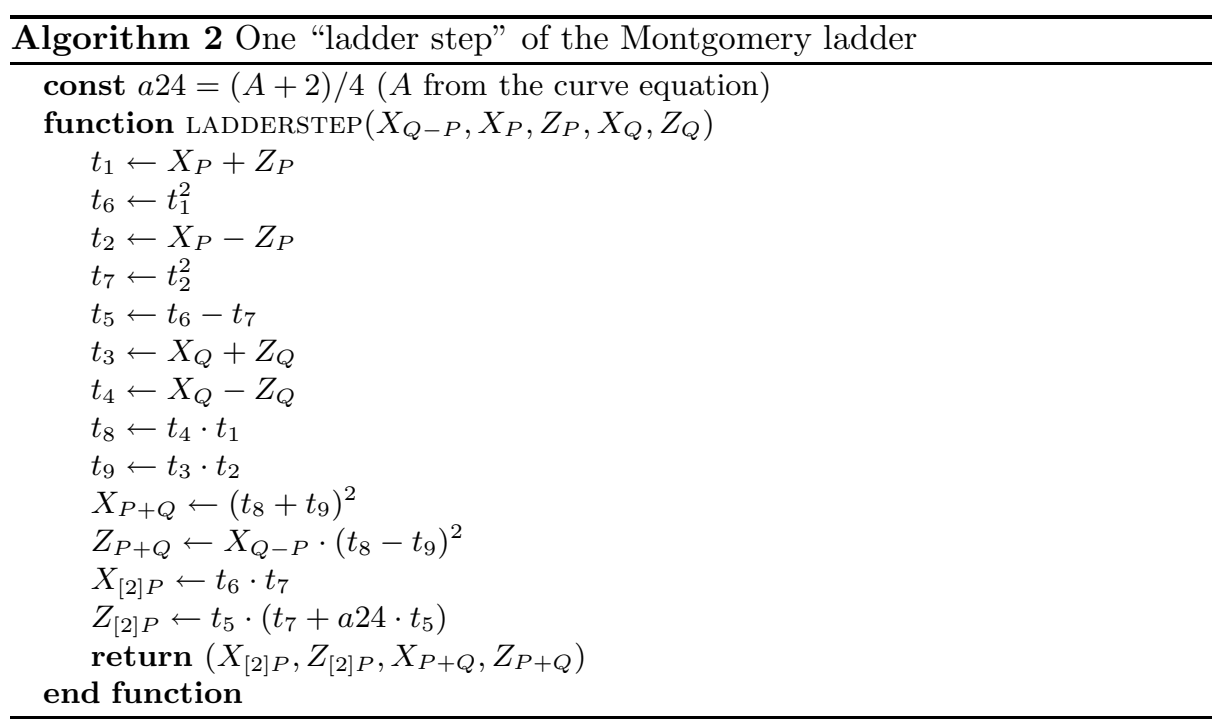

gives as output a 32-byte string representing the $x$-coordinate $x_{Q}$ of $Q=[k] P$. For each of these values curve25519 is assuming little-endian representation.

For our implementation we decided to follow [3] and compute $x_{Q}$ by first using Algorithm 1 to compute $\left(X_{Q}, Z_{Q}\right)$ and then computing $x_{Q}=Z_{Q}^{-1} \cdot X_{Q}$.

\section{The MPM library and ECC}

\subsection{Implementation hierarchy}

Implementations of elliptic-curve cryptography are often described as a hierarchy of operations. In [10, Section 5.2.1] Hankerson, Menezes and Vanstone outline a hierarchy of operations in ECC as protocols, point multiplication, elliptic-curve addition and doubling, finite-field arithmetic. Fan, Sakiyama \& Verbauwhede expand this in [7] to describe a 5-layer pyramid of

1. Integrity, confidentially, authentication,

2. Elliptic-curve scalar multiplication $[k] P$,

3. Point addition and doubling,

4. Modular operations in $\mathbb{F}_{p}$,

5. Instructions of a $w$-bit core.

\section{2 $\quad \mathbb{F}_{p}$ arithmetic using the MPM library}

In Section 3 we described how the upper 3 layers of this hierarchy are handled. Hence, the obvious next step is to look at efficient modular operations in $\mathbb{F}_{p}$ and how these operations can be mapped to the SIMD instructions on 128-bit registers of the SPU. 
For this task of mapping operations on large integers to the SPU instruction set, IBM provides a vector-optimized multi-precision math (MPM) library [14] as part of the software development kit (SDK) offered for the Cell Broadband Engine.

This MPM library is provided in source code and its algorithms are generic for arbitrary sized numbers. They operate on 16-bit halfwords as smallest units, elements of our 255-bit field are therefore actually handled as 256-bit values.

As our computation is mostly bottlenecked by costs for multiplications and squarings in the finite field we decided to optimize these functions (classic and Montgomery method) for 256-bit input values.

At a high level the original MPM multiplication functions are structured by an array declaration section, then array initialization via loop dictated by the input sizes, a main body consisting of calculating partial products inside nested loops (again determined by the input sizes), and finally a gather section where the partial products feed into the result.

We optimized this pattern for fixed 256-bit inputs by using the large register array to remove the implicit array calls and then fully unrolled the loops. The Montgomery routine also lets us use a __builtin_expect compiler directive at the final overflow test to direct the hardware to which branch will be taken and avoid an expensive stall. While these manual unroll and branch hint techniques help both the GCC and IBM XLC compilers it should be noted that the GCCderived compiler achieves a $10 \%$ improvement over the XLC compiler ${ }^{3}$

The MPM library supplies a specialized function for squaring where significant optimizations should be made over a general multiply by reusing partial products. However our timings indicate that such savings are not achieved until the size of the multi-precision inputs exceeds 512-bits. We therefore take the timings of a multiplication for a squaring.

\subsection{What speed can we achieve using MPM?}

The Montgomery ladder in the curve25519 computation consists of 255 ladder steps, hence, computation takes 1276 multiplications, 1020 squarings, 255 multiplications with a constant, 2040 additions and one inversion in the finite field $\mathbb{F}_{2^{255}-19}$. Table 1 gives the number of CPU cycles required for each of these operations (except inversion).

For finite field multiplication and squaring we benchmarked two possibilities: a call to mpm_mul followed by a call to mpm_mod and the Montgomery multiplication function mpm_mont_mod_mul. Addition is implemented as a call to mpm_add and a conditional call (mpm_cmpge) to mpm_sub. For multiplication we include timings of the original MPM functions and of our optimized versions. The original MPM library offers a number of options for each operation. We select the inlined option with equal input sizes for fair comparison.

From these numbers we can compute a lower bound of 2227040 cycles (1276M $+1020 \mathrm{~S}+2040 \mathrm{~A}$, where M, S and A stand for the costs of multiplication,

\footnotetext{
${ }^{3}$ IBM XL C $/ C++$ for Multicore Acceleration for Linux, V10.1. CBE SDK 3.1
} 


\begin{tabular}{|l|r|}
\hline Operation & Number of cycles \\
\hline \hline Addition/Subtraction & 86 \\
\hline Multiplication (original MPM) & 4334 \\
\hline Multiplication (optimized) & 4124 \\
\hline Montgomery Multiplication (original MPM) & 1197 \\
\hline Montgomery Multiplication (optimized) & 892 \\
\hline
\end{tabular}

Table 1. MPM performance for arithmetic operations in a 256-bit finite field

squaring and addition respectively) required for the curve25519 computation when using MPM. Observe that this lower bound still ignores costs for the inversion and for multiplication with the constant.

The high cost for modular reduction in these algorithms results from the fact, that the MPM library cannot make use of the special form of the modulus $2^{255}-19$; an improved, specialized reduction routine would probably yield a smaller lower bound. We therefore investigate what lower bound we get when entirely ignoring costs for modular reduction. Table 2 gives numbers of cycles for multiplication and addition of 256-bit integers without modular reduction. This yields a lower bound of 934080 cycles. Any real implementation would, of course, take significantly more time as it would need to account for operations not considered in this estimation.

\begin{tabular}{|l|r|}
\hline Operation & Number of cycles \\
\hline \hline Addition/Subtraction & 52 \\
\hline Multiplication (original MPM) & 594 \\
\hline Multiplication (optimized) & 360 \\
\hline
\end{tabular}

Table 2. MPM performance for arithmetic operations on 256-bit integers

\section{Implementation of curve25519}

As described in Section 3 the computation of the curve25519 function consists of two parts, the Montgomery ladder computing $\left(X_{Q}, Z_{Q}\right)$ and the inversion of $Z_{Q}$.

We decided to implement the inversion as an exponentiation with $p-2=$ $2^{255}-21$ using the the same sequence of 254 squarings and 11 multiplications as [3]. This might not be the most efficient algorithm for inversion, but it is the easiest way to implement an inversion algorithm which takes constant time independent of the input. 
Completely inlining all multiplications and squarings would result in an excessive increase of the overall code size. We therefore implement multiplication and squaring functions and use calls to these functions.

However for the first part - the Montgomery ladder-we do not use calls to these functions but take one ladder step as smallest unit implemented in a function. This allows for a higher degree of data-level parallelism, especially in the modular reductions, and thus yields a significantly increased performance.

For the speed-critical parts of our implementation we use the qhasm programming language [2], which offers us all flexibility for code optimization on the assembly level, while still supporting a more convenient programming model than plain assembly. We extended this language to also support the SPU of the Cell Broadband Engine as target architecture.

In the description of our implementation we will use the term "register variable". Note that for qhasm (unlike C) the term register variable refers to variables that are forced to be kept in registers.

\subsection{Fast arithmetic}

In the following we will first describe how we represent elements of the finite field $\mathbb{F}_{2^{255}-19}$ and then detail the three algorithms that influence execution speed of curve25519 most, namely finite field multiplications, finite field squaring and a Montgomery ladder step.

\subsection{Representing elements of $\mathbb{F}_{2^{255}-19}$}

We represent an element $a$ of $\mathbb{F}_{2^{255}-19}$ as a tuple $\left(a_{0}, \ldots, a_{19}\right)$ where

$$
a=\sum_{i=0}^{19} a_{i} 2^{\lceil 12.75 i\rceil} .
$$

We call a coefficient $a_{i}$ reduced if $a_{i} \in\left[0,2^{13}-1\right]$. Analogously we call the representation of an element $a \in \mathbb{F}_{2^{255}-19}$ reduced if all its coefficients $a_{0}, \ldots, a_{19}$ are reduced.

As described in section 2 the Cell Broadband Engine can only perform 16-bit integer multiplication, where one instruction performs 4 such multiplications in parallel. In order to achieve high performance of finite field arithmetic it is crucial to properly arrange the values $a_{0}, \ldots a_{19}$ in registers and to adapt algorithms for field arithmetic to make use of this SIMD capability.

Multiplication and Squaring in $\mathbb{F}_{\mathbf{2}^{255}-19}$ As input to field multiplication we get two finite field elements $\left(a_{0}, \ldots, a_{19}\right)$ and $\left(b_{0}, \ldots, b_{19}\right)$. We assume that these field elements are in reduced representation. This input is arranged in 10 register variables a03, a47, a811, a1215, a1619, b03, b47, b811, b1215 and b1619 as follows: Register variable a03 contains in its word elements the coefficients 
$a_{0}, a_{1}, a_{2}, a_{3}$, register variable a47 contains in its word elements the coefficients $a_{4}, a_{5}, a_{6}, a_{7}$, and so on.

The idea of multiplication is to compute coefficients $r_{0}, \ldots, r_{38}$ of $r=a b$ where:

$$
\begin{aligned}
& r_{0}=a_{0} b_{0} \\
& r_{1}=a_{1} b_{0}+a_{0} b_{1} \\
& r_{2}=a_{2} b_{0}+a_{1} b_{1}+a_{0} b_{2} \\
& r_{3}=a_{3} b_{0}+a_{2} b_{1}+a_{1} b_{2}+a_{0} b_{3} \\
& r_{4}=a_{4} b_{0}+2 a_{3} b_{1}+2 a_{2} b_{2}+2 a_{1} b_{3}+a_{0} b_{4} \\
& r_{5}=a_{5} b_{0}+a_{4} b_{1}+2 a_{3} b_{2}+2 a_{2} b_{3}+a_{1} b_{4}+a_{0} b_{5} \\
& r_{6}=a_{6} b_{0}+a_{5} b_{1}+a_{4} b_{2}+2 a_{3} b_{3}+a_{2} b_{4}+a_{1} b_{5}+a_{0} b_{6} \\
& r_{7}=a_{7} b_{0}+a_{6} b_{1}+a_{5} b_{2}+a_{4} b_{3}+a_{3} b_{4}+a_{2} b_{5}+a_{1} b_{6}+a_{0} b_{7} \\
& r_{8}=a_{8} b_{0}+2 a_{7} b_{1}+2 a_{6} b_{2}+2 a_{5} b_{3}+a_{4} b_{4}+2 a_{3} b_{5}+2 a_{2} b_{6}+2 a_{1} b_{7}+a_{0} b_{8}
\end{aligned}
$$

This computation requires 400 multiplications and 361 additions. Making use of the SIMD instructions, at best 4 of these multiplications can be done in parallel, adding the result of a multiplication is at best for free using the mpya instruction, so we need at least 100 instructions to compute the coefficients $r_{0}, \ldots, r_{38}$. Furthermore we need to multiply some intermediate products by 2 , an effect resulting from the non-integer radix 12.75 used for the representation of finite field elements. As we assume the inputs to have reduced coefficients, all result coefficients $r_{i}$ fit into 32 -bit word elements.

We will now describe how the coefficients $r 0, \ldots, r 38$ can be computed using 145 pipeline-0 instructions (arithmetic instructions). This computation requires some rearrangement of coefficients in registers using the shufb instruction but with careful instruction scheduling and alignment these pipeline- 1 instructions do not increase the number of cycles needed for multiplication. From the description of the arithmetic instructions it should be clear which rearrangement of inputs is necessary.

First use $15 \mathrm{shl}$ instructions to have register variables b03s 1 containing $b_{0}, b_{1}, b_{2}, 2 b_{3}$, b03s 2 containing $b_{0}, b_{1}, 2 b_{2}, 2 b_{3}$, b03s3 containing $b_{0}, 2 b_{1}, 2 b_{2}, 2 b_{3}$, b47s 1 containing $b_{4}, b_{5}, b_{6}, 2 b_{7}$ and so on.

Now we can proceed producing intermediate result variables r03 containing $a_{0} b_{0}, a_{0} b_{1}, a_{0} b_{2}, a_{0} b_{3}$ (one mpy instruction), r14 containing $a_{1} b_{0}, a_{1} b_{1}, a_{1} b_{2}, 2 a_{1} b_{3}$ (one mpy instruction), r25 containing $a_{2} b_{0}, a_{2} b_{1}, 2 a_{2} b_{2}, 2 a_{2} b_{3}$ (one mpy instruction), r36 containing $a_{3} b_{0}, 2 a_{3} b_{1}, 2 a_{3} b_{2}, 2 a_{3} b_{3}$ (one mpy instruction), r47 containing $a_{4} b_{0}+a_{0} b_{4}, a_{4} b_{1}+a_{0} b_{5}, a_{4} b_{2}+a_{0} b_{6}, a_{4} b_{3}+a_{0} b_{7}$ (one mpy and one mpya instruction), 
r58 containing $a_{5} b_{0}+a_{1} b_{4}, a_{5} b_{1}+a_{1} b_{5}, a_{5} b_{2}+a_{1} b_{6}, 2 a_{5} b_{3}+2 a_{1} b_{7}$ (one mpy and one mpya instruction) and so on. In total these computations need 36 mpy and 64 mpya instructions.

As a final step these intermediate results have to be joined to produce the coefficients $r_{0}, \ldots r_{38}$ in the register variables $r 03, r 47, \ldots r 3639$. We can do this using 30 additions if we first combine intermediate results using the shufb instruction. For example we join in one register variable the highest word of $r 14$ and the three lowest words of r58 before adding this register variable to $r 47$.

The basic idea for squaring is the same as for multiplication. We can make squaring slightly more efficient by exploiting the fact that some intermediate results are equal.

For a squaring of a value $a$ given in reduced representation $\left(a_{0}, \ldots, a_{19}\right)$, formulas for the result coefficients $r_{0}, \ldots, r_{38}$ are the following:

$$
\begin{aligned}
& r_{0}=a_{0} a_{0} \\
& r_{1}=2 a_{1} a_{0} \\
& r_{2}=2 a_{2} a_{0}+a_{1} a_{1} \\
& r_{3}=2 a_{3} a_{0}+2 a_{2} a_{1} \\
& r_{4}=2 a_{4} a_{0}+4 a_{3} a_{1}+2 a_{2} a_{2} \\
& r_{5}=2 a_{5} a_{0}+2 a_{4} a_{1}+4 a_{3} a_{2} \\
& r_{6}=2 a_{6} a_{0}+2 a_{5} a_{1}+2 a_{4} a_{2}+2 a_{3} a_{3} \\
& r_{7}=2 a_{7} a_{0}+2 a_{6} a_{1}+2 a_{5} a_{2}+2 a_{4} a_{3} \\
& r_{8}=2 a_{8} a_{0}+4 a_{7} a_{1}+4 a_{6} a_{2}+4 a_{5} a_{3}+a_{4} a_{4}
\end{aligned}
$$

The main part of the computation only requires 60 multiplications (24 mpya and 36 mpy instructions). However, some partial results have to be multiplied by 4 ; this requires more preprocessing of the inputs, we end up using 35 instead of $15 \mathrm{shl}$ instructions before entering the main block of multiplications. Squaring is therefore only 20 cycles faster than multiplication.

During both multiplication and squaring, we can overcome latencies by interleaving independent instructions.

\subsection{Reduction}

The task of the reduction step is to compute from the coefficients $r_{0}, \ldots r_{38}$ a reduced representation $\left(r_{0}, \ldots, r_{19}\right)$. Implementing this computation efficiently is challenging in two ways: In a typical reduction chain every instruction is dependent on the result of the preceding instruction. This makes it very hard to vectorize operations in SIMD instructions and to hide latencies.

We will now describe a way to handle reduction hiding most instruction latencies but without data level parallelism through SIMD instructions. 
The basic idea of reduction is to first reduce the coefficients $r_{20}$ to $r_{38}$ (producing a coefficient $r_{39}$ ), then add $19 r_{20}$ to $r_{0}, 19 r_{21}$ to $r_{1}$ and so on until adding $19 r_{39}$ to $r_{19}$ and then reduce the coefficients $r_{0}$ to $r_{19}$.

Multiplications by 19 result from the fact, that the coefficient $a_{20}$ stands for $a_{20} \cdot 2^{255}$ (see equation (1)). By the definition of the finite field $\mathbb{F}_{2^{255}-19}, 2^{255} a_{20}$ is the same as $19 a_{20}$. Equivalent statements hold for the coefficients $a_{21}, \ldots, a_{39}$.

The most speed critical parts of this reduction are the two carry chains from $r_{20}$ to $r_{39}$ and from $r_{0}$ to $r_{19}$. In order to overcome latencies in these chains we break each of them into four parallel carry chains, Algorithm 3 describes this structure of our modular reduction algorithm.

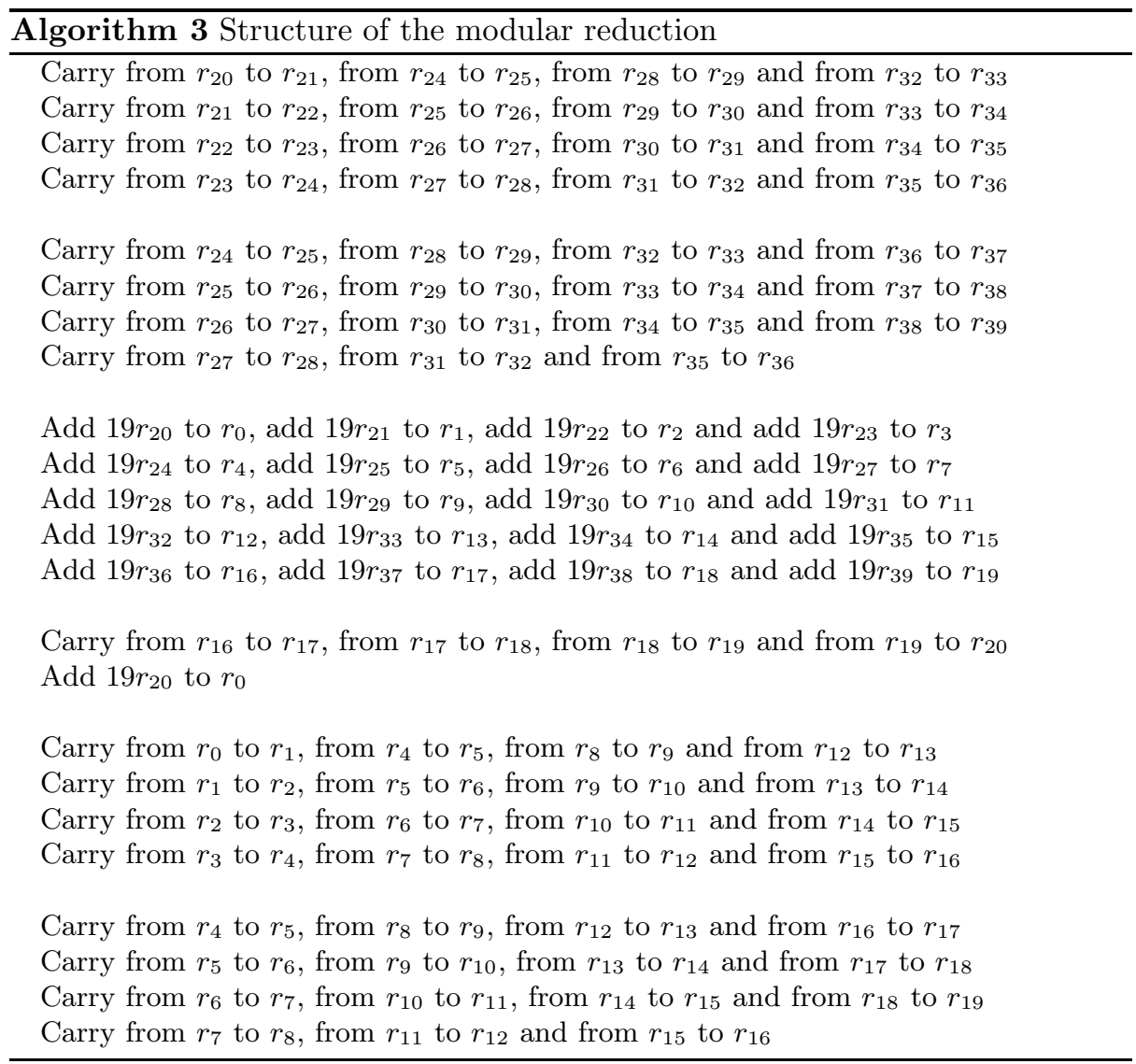

Each of the carry operations in Algorithm 3 can be done using one shufb, one rotmi and one a instruction. Furthermore we need 8 masking instructions (bitwise and) for each of the two carry chains.

In total, a call to the multiplication function (including reduction) takes 444 cycles, a call to the squaring function takes 424 cycles. This includes 144 cycles 
for multiplication (124 cycles for squaring), 244 cycles for reduction and some more cycles to load input and store output. Furthermore the cost of a function call is included in these numbers.

Montgomery ladder step For the implementation of a Montgomery ladder step we exploit the fact that we can optimize a fixed sequence of arithmetic instructions in $\mathbb{F}_{2^{255}-19}$ instead of single instructions. This makes it much easier to make efficient use of the SIMD instruction set, in particular, for modular reduction.

The idea is to arrange the operations in $\mathbb{F}_{2^{255}-19}$ into blocks of 4 equal or similar instructions, similar meaning that multiplications and squarings can be grouped together and additions and subtractions can be grouped together as well. Then these operations can be carried out using the 4-way parallel SIMD instructions in the obvious way; for example for 4 multiplications $r=a \cdot b$, $s=c \cdot d, t=e \cdot f$ and $u=g \cdot h$ we first produce register variables aceg0 containing in its word elements $a_{0}, c_{0}, e_{0}, g_{0}$ and bdgho containing $b_{0}, d_{0}, e_{0}, g_{0}$ and so on. Then the first coefficient of $r, s, t$ and $u$ can be computed by applying the mpy instruction on aceg0 and bdfh0. All other result coefficients of $r, s, t$ and $u$ can be computed in a similar way using mpy and mpya instructions.

This way of using the SIMD capabilities not only makes multiplication slightly faster (420 arithmetic instructions instead of 576 for 4 multiplications), it also allows for much faster reduction: The reduction algorithm described above can now be applied to 4 results in parallel, reducing the cost of a reduction by a factor of 4 .

In Algorithm 4 we describe how we divide a Montgomery ladder step into blocks of 4 similar operations. In this algorithm the computation of $Z_{P+Q}$ in the last step requires one multiplication and reduction which we carry out as described in the previous section. The computation of a ladder step again requires rearrangement of data in registers using the shufb instruction. Again we can hide these pipeline- 1 instructions almost entirely by interleaving with arithmetic pipeline-0 instructions.

One remark regarding subtractions occurring in this computation: As reduction expects all coefficients to be larger than zero, we cannot just compute the difference of each coefficient. Instead, for the subtraction $a-b$ we first add $2 p$ to $a$ and then subtract $b$. For blocks containing additions and subtractions in Algorithm 4 we compute the additions together with additions of $2 p$ and perform the subtraction in a separate step.

In total one call to the ladder-step function takes 2433 cycles.

\section{Results and Comparison}

\subsection{Benchmarking Methodology}

In order to make our benchmarking results comparable and verifiable we use the SUPERCOP toolkit, a benchmarking framework developed within eBACS, 


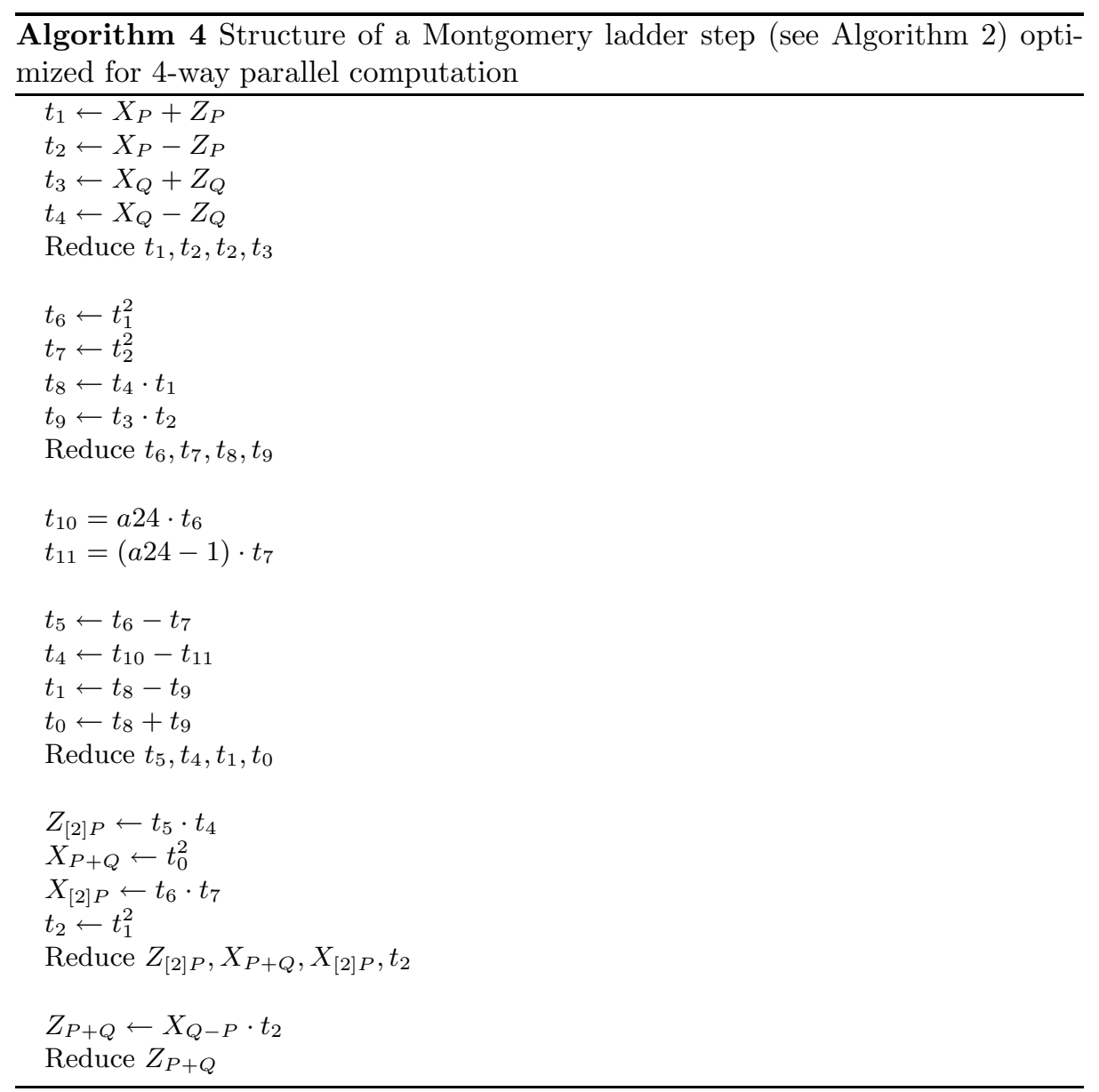

the benchmarking project of ECRYPT II [4]. The software presented in this paper passes the extensive tests of this toolkit showing compatibility to other curve25519 implementations, in particular the reference implementation included in the toolkit.

For scalar multiplication software, SUPERCOP measures two different cycle counts: The crypto_scalarmult benchmark measures cycles for a scalar multiplication of an arbitrary point; the crypto_scalarmult_base benchmark measures cycles needed for a scalar multiplication of a fixed base point.

We currently implement crypto_scalarmult_base as crypto_scalarmult; faster implementations would be useful in applications that frequently call crypto_scalarmult_base

Two further benchmarks regard our curve25519 software in the context of Diffie-Hellman key exchange: The crypto_dh_keypair benchmark measures the number of cycles to generate a key pair consisting of a secret and a public key. 
The crypto_dh benchmark measures cycles to compute a joint key, given a secret and a public key.

\subsection{Results}

We benchmarked our software on a computer named hex01, a QS21 blade at the Chair for Operating Systems at RWTH Aachen University. It contains two $3200 \mathrm{MHz}$ Cell Broadband Engine processors (revision 5.1); measurements used one SPU of one CBE:

\begin{tabular}{|l|r|}
\hline SUPERCOP benchmark on hex01 & number of cycles \\
\hline \hline crypto_scalarmult & 777000 \\
\hline crypto_scalarmult_base & 777000 \\
\hline crypto_dh_keypair & 804000 \\
\hline crypto_dh & 780720 \\
\hline
\end{tabular}

We furthermore benchmarked the software on a computer named node033, a QS22 blade at the Research Center Jülich. It contains two PowerXCell 8i processors (Cell Broadband Engine (revision 48.0)); measurements used one SPU of one CBE:

\begin{tabular}{|l|r|}
\hline SUPERCOP benchmark on node033 & number of cycles \\
\hline \hline crypto_scalarmult & 777000 \\
\hline crypto_scalarmult_base & 777000 \\
\hline crypto_dh_keypair & 804000 \\
\hline crypto_dh & 780840 \\
\hline
\end{tabular}

We also benchmarked our software on a computer named cosmovoid, a Sony Playstation 3 at the Chair for Operating Systems at RWTH Aachen University. It has a $3192 \mathrm{MHz}$ Cell Broadband Engine processor (revision 5.1); measurements used one SPU of this CBE.

\begin{tabular}{|l|r|}
\hline SUPERCOP benchmark on cosmovoid & number of cycles \\
\hline \hline crypto_scalarmult & 777040 \\
\hline crypto_scalarmult_base & 777040 \\
\hline crypto_dh_keypair & 803960 \\
\hline crypto_dh & 780720 \\
\hline
\end{tabular}

\subsection{Comparison}

To compare our results on a cost-performance basis with ECDH software for other platforms, we consider the cheapest hardware configuration containing a Cell Broadband Engine, namely the Sony Playstation 3, and compare the results to an Intel-Core-2-based configuration running the ECDH software presented in [8]. This is the currently fastest implementation of ECDH for the Core 2 
processor providing a similar security as curve25519. Note that this software is not protected against timing attacks.

We benchmarked this software on a machine called archer. at the National Taiwan University. This machine has a $2500 \mathrm{MHz}$ Intel Core 2 Q9300 processor with 4 cores; measurements used one core.

SUPERCOP reports 365363 cycles for the crypto_dh benchmark (this software is not benchmarked as scalar-multiplication software). Key-pair generation specializes the scalar multiplication algorithm for the known basepoint; the crypto_dh_keypair benchmark reports 151215 cycles.

For the Core-2-based configuration we determined which processor (as part of a workstation) yields the best cost-performance ratio. Currently this is the Q9300, a quad-core processor operating at $2.5 \mathrm{GHz}$. To estimate a price for a complete workstation including this processor we determined the lowest prices for processor, case, motherboard, memory, hard disk and power supply from the online retailer zipzoomfly.com yielding $\$ 402.66$.

To determine the best price for the Sony Playstation 3 we used Google Product Search. The currently (Jan 6, 2009) cheapest offer is $\$ 279$ for the Playstation 3 with a 40 GB hard disk.

The Sony Playstation 3 makes 6 SPUs available for general purpose computations. Using our implementation running at 780720 cycles (crypto_dh on cosmovoid) on 6 SPUs operating at $3192 \mathrm{MHz}$ yields 24528 curve25519 computations per second. Taking the $\$ 279$ market price for the Playstation as a basis, the cheapest CBE-based hardware can thus perform 87 computations of curve25519 per second per dollar.

The Q9300-based workstation has 4 cores operating at $2.5 \mathrm{GHz}$, using the above-mentioned implementation which takes 365363 cycles, we can thus perform 27368 joint-key computations per second. Taking $\$ 400$ market price for a Q9300-based workstation as a basis, the cheapest Core-2-based hardware can thus perform 68 joint-key computations per second per dollar.

Note, that this comparison is not fair in several ways: The cheapest Q9300based workstation has for example more memory than the Playstation 3 (1GB instead of $256 \mathrm{MB}$ ) and a larger hard disk (160GB instead of 40GB).

On the other hand we only use the 6 SPUs of the CBE for the curve25519 computation, the PPU is still available for other tasks, whereas the performance estimation for the Core-2-based system assumes $100 \%$ workload on all CPU cores.

In any case it is clear that the Cell Broadband Engine, when used properly, is one of the best available CPUs for public-key cryptography.

\section{References}

1. Wesley Alvaro, Jakub Kurzak, and Jack Dongarra. Fast and small short vector SIMD matrix multiplication kernels for the synergistic processing element of the CELL processor. In Computational Science - ICCS 2008), volume 5101 of Lecture Notes in Computer Science, pages 935-944. Springer, 2008. 
2. Daniel J. Bernstein. qhasm: tools to help write high-speed software. http://cr . yp.to/qhasm.html (accessed Jan 1, 2009).

3. Daniel J. Bernstein. Curve25519: new Diffie-Hellman speed records. In Public Key Cryptography - PKC 2006, volume 3958 of Lecture Notes in Computer Science, pages 207-228. Springer, 2005.

4. Daniel J. Bernstein and Tanja Lange (editors). eBACS: ECRYPT benchmarking of cryptographic systems, Nov 2008. http://bench.cr.yp.to/ (accessed Jan 1, 2009).

5. Neil Costigan and Michael Scott. Accelerating SSL using the vector processors in IBM's Cell Broadband Engine for Sony's Playstation 3. In Proceedings of SPEED workshop, 2007. http://www.hyperelliptic.org/SPEED/.

6. Whitfield Diffie and Martin E. Hellman. New directions in cryptography. IEEE Transactions on Information Theory, IT-22(6):644-654, Nov 1976. http:// citeseer.ist.psu.edu/diffie76new.html.

7. Junfeng Fan, Kazuo Sakiyama, and Ingrid Verbauwhede. Elliptic curve cryptography on embedded multicore systems. In Workshop on Embedded Systems Security - WESS 200\%, pages 17-22, Salzburg,Austria, 2007.

8. Stephen D. Galbraith, Xibin Lin, and Michael Scott. Endomorphisms for faster elliptic curve cryptography on general curves, 2008. http://eprint.iacr.org/ 2008/194.

9. P. Gaudry and E. Thomé. The $\mathrm{mpF}_{q}$ library and implementing curve-based key exchanges. In Proceedings of SPEED workshop, 2007. http://www.loria.fr/ $\sim$ gaudry/publis/mpfq.pdf.

10. Darrel Hankerson, Alfred J. Menezes, and Scott A. Vanstone. Guide to Elliptic Curve Cryptography. Springer, Berlin, 2003.

11. IBM DeveloperWorks. Cell broadband engine architecture and its first implementation, Nov 2005. http://www-128.ibm.com/developerworks/power/library/ pa-cellperf /.

12. IBM DeveloperWorks. Cell broadband engine programming handbook (version 1.1), April 2007. http://www-01.ibm.com/chips/techlib/techlib.nsf/ techdocs/9F820A5FFA3ECE8C8725716A0062585F.

13. IBM DeveloperWorks. SPU assembly language specification (version 1.6), Sep 2007. http://www-01.ibm.com/chips/techlib/techlib.nsf/techdocs/ EFA2B196893B550787257060006FC9FB.

14. IBM DeveloperWorks. Example library API reference (version 3.1), Sep 2008. http://www.ibm.com/developerworks/power/cell/documents.html.

15. IBM DeveloperWorks. SPE cryptographic library user documentation 1.0, Sep 2008. http://www.ibm.com/developerworks/power/cell/documents.html.

16. Peter. L. Montgomery. Speeding the Pollard and elliptic curve methods of factorization. Mathematics of Computation, 48(177):243-264, 1987.

17. Kanna Shimizu, Daniel Brokenshire, and Mohammad Peyravian. Cell Broadband Engine support for privacy, security, and digital rights management applications. White paper, IBM, Oct 2005. http://www-01.ibm.com/chips/techlib/techlib. nsf/techdocs/3F88DA69A1C0AC40872570AB00570985.

18. Alexander Sotirov, Marc Stevens, Jacob Appelbaum, Arjen Lenstra, David Molnar, Dag Arne Osvik, and Benne de Weger. MD5 considered harmful today, Dec 2008. http://www.win.tue.nl/hashclash/rogue-ca/ (accessed Jan 4, 2009).

19. Marc Stevens, Arjen Lenstra, and Benne de Weger. Nostradamus - predicting the winner of the 2008 US presidential elections using a Sony PlayStation 3, Nov 2007. http://www.win.tue.nl/hashclash/Nostradamus/ (accessed Jan 4, 2009). 